\title{
Resistive Switching Studies of ReRAM Devices by In-Situ TEM
}

$\underline{\text { Gemma Martín }}^{1}$, Mireia B. González ${ }^{2}$, Aïda Varea ${ }^{1}$, Oriol Blázquez ${ }^{1}$, Giovanni Vescio ${ }^{1}$, Francesca Campabadal $^{2}$, Sergi Hernández ${ }^{1}$, Albert Cirera ${ }^{1}$, Blas Garrido ${ }^{1}$, Sònia Estradé ${ }^{1}$, Francesca Peiró ${ }^{1}$ and Albert Cornet ${ }^{1}$

${ }^{1 .}$ MIND/IN2UB, Departament d'Enginyeria Electrònica i Biomèdica, Universitat de Barcelona, Martí i Franquès 1, 08028 Barcelona, Spain.

2. Institut de Microelectrònica de Barcelona, IMB-CNM (CSIC), Campus UAB, 08193 Bellaterra, Spain.

Resistive random access memory (ReRAM) devices have been extensively researched in recent years to address scaling issues in nonvolatile flash memories, which are based in charge-trapping. The most critical problem for the development of ReRAM technology is the detailed understanding of both the mechanisms of conductive filament (CF) formation and switching mechanism. The nanometric nature of the switching region and its random location in the device make it extremely difficult to characterize.

In this sense, in-situ TEM has been demonstrated as a valuable tool to explore ReRAMs switching mechanisms. D.H. Kwon et al. [1] demonstrate with in-situ current-voltage and HRTEM studies that switching occurs by the formation and disruption of $\mathrm{Ti}_{\mathrm{n}} \mathrm{O}_{2 \mathrm{n}-1}$ filaments of Magnéli phase. Q. Liu et al. [2] describe, using in-situ TEM, in $\mathrm{ZrO}_{2}$ based ReRAM devices, real-time observations of the $\mathrm{CF}$ formation and dissolution processes mechanism based on the local redox reaction inside the $\mathrm{ZrO}_{2}$-electrolyte system. Besides, $\mathrm{X}$. Wu et al. [3][4] observe the formation and dissolution of nanofilaments in $\mathrm{HfO}_{2}$ based ReRAM devices by TEM in real time, observing oxygen ion drift and the subsequent Ni migration into the dielectric and the substrate from the top electrode.

In this work, the structural and chemical modifications of three different ReRAM systems are analyzed at the nanoscale after CF formation by means of HRTEM, EELS and EDX. The employed systems consist of: (1) silicon-aluminum oxynitride (SiAlON) thin film, grown by pulsed-laser deposition (PLD) [5]; (2) a $\mathrm{HfO}_{2}$-based ReRAM inkjet-printed structure; and (3) a Ni/HfO $/ \mathrm{H}_{2} / \mathrm{Si}$ structure with the $\mathrm{HfO}_{2}$ film, grown by atomic layer deposition (ALD) [6].

Moreover, in-situ biasing TEM experiments are performed to observe the formation of the CF in real time and to understand the physical mechanism behind the CF formation in ReRAM devices.

\section{References:}

[1] Deok-Hwang Kwon et al, Nature Nanotechnology 5 (2010), p. 148.

[2] Qi Liu et al, Adv Mater 24 (2012), p. 1844.

[3] Xing Wu et al, Adv Electron Mater 1 (2015), p. 11.

[4] Xing Wu et al, J Appl Phys 113 (2013), p. 114503.

[5] Oriol Blázquez et al, Nanotechnology 29 (2018), p. 235702.

[6] Gemma Martín et al, Appl Phys Express 11 (2018), p. 014101. 


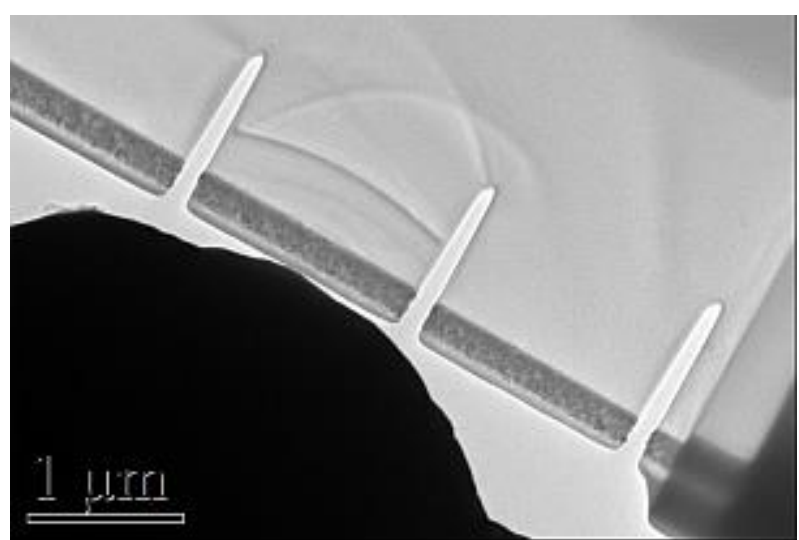

Figure 1. TEM image of the STM tip contacting the $\mathrm{Ni} / \mathrm{HfO}_{2} / \mathrm{Si}$ ReRAM device 